\title{
SCIDoc

\section{Transconjunctival Lower Lid Blepharoplasty Made Easy by Everting the Eyelid}

Peter PM Raus*

Miró Center for Ophthalmology and Aesthetics, Geel, Belgium.

\section{Abstract}

Purpose: To evaluate the transconjunctival lower lid blepharoplasty using a Desmarres lid retractor to evert the eyelid. Design: Prospective study.

Methods: Twenty-one cases of inferior blepharoplasty in 11 patients, performed through a transconjunctival approach are reported. The author describes the surgical technique with Radio Frequency surgery, the use of a Desmarres lid retractor to evert the eyelid and the results.

Results: Radio Frequency surgery and the use of a Desmarres lid retractor appear to give excellent results in transconjunctival lower lid blepharoplasty.

Everting instead of retracting the eyelid provides excellent access to the fat pockets in the lower lids with less bleeding thanks to the traction on the eyelid.

The transconjunctival approach has a lower complication rate than the classic transcutaneous approach, with no visible scar and less risk of postoperative scleral show or ectropion.

Conclusion: Radiosurgically assisted transconjunctival blepharoplasty of the lower lid with the use of a Desmarres lid retractor is associated with a good functional and cosmetic outcome.

Keywords: Transconjunctival Approach; Radio Frequency Surgery; Desmarres; Lower Lid Blepharoplasty.

\section{Introduction}

A prolapse of orbital fat in the lower eyelid is the most frequent reason for patients to ask for a lower bleph. In the lower lid only in very rare cases a resection of skin is needed and that is why in the lower lid the transconjunctival blepharoplasty is out favorite approach. Also, in transconjunctival blephs there is no visible scar. No sutures have to be placed (or removed) and the orbicularis muscle does not need to be incised [1]. For this reason the risk for postoperative ectropion and/or scleral show is minimal. Indeed: not only excessive skin resection can cause ectropion. Even a simple incision in the orbicularis muscle-especially in the pretarsal part when making a subciliary incision-or the orbital septum can pull the eyelid down (scleral show) or out (ectropion) as it heals [7]. This complication can be very difficult to correct [2]. Also, an incision in the highly vascularized tarsal conjunctiva can induce a significant bleeding, especially in patients with a short lower eyelid and shallow cul de sac where the fat pockets are not easily accessible [3]. Our technique of transconjunctival blephs of the lower eyelid tries to deal with all these aspects.

\section{Materials and Methods}

\section{Anesthesia}

Even with surgery under general anesthesia, we start with a subcutaneous injection of Xylocaïne $2 \%$ with epinephrine. Then the lower eyelid is everted using a Desmarres lid retractor and subsequently Xylocaïne $2 \%$ with epinephrine is injected subconjunctivally. The purpose of a local anesthesia both from the outside as transconjunctivally is fourfold:

- Additional anesthesia (in patients under local anesthesia).

- Vasoconstriction by epinephrine.

- Hydrodissection of the conjunctiva and the retractor muscle.

- Wetting the tissues with the injection of anesthetic facilitates the radiosurgical incision.

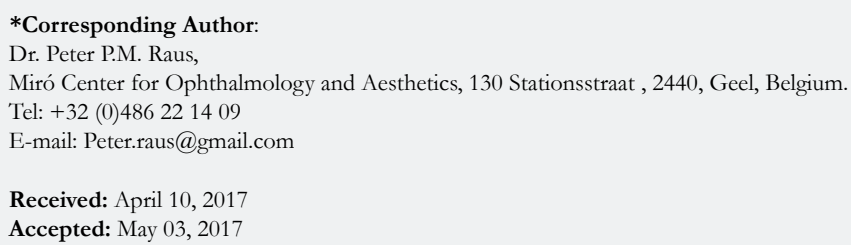

Copyright: Peter PM Raus ${ }^{\circ}$ 2017. This is an open-access article distributed under the terms of the Creative Commons Attribution License, which permits unrestricted use, distribution and reproduction in any medium, provided the original author and source are credited. 
Although theoretically surgery can be performed both under local and general anesthesia, we prefer the latter: the transconjunctival incision appears to be more stressful for severel patients. This can induce the blood pressure to rise with more bleeding. An intervention under general anesthesia therefore is not only safer but also more comfortable for both surgeon and patient. Besides, in dialogue with the anesthesiologist blood pressure can be lowered maximally during general anesthesia.

\section{Surgical Technique}

The lower lid is not retracted but everted wit a Desmarres lid retractor. Additional Xylocaïne 2\% with epinephrine is injected subconjunctivally and as superficially as possible. Thanks to both the stretching of the lower eyelid with the lid retractor and the effect of the epinephrine an almost bloodless area is created to further diminish the bleeding during the intervention. The stretching of the lid also facilitates the identification of the lower tarsal border. Also, the incision of the lower lid layer by layer becomes much easier. For the radiosurgical incision in the conjunctiva we use a relatively thick electrode with the unit in the cut/coagulate mode which gives $50 \%$ cutting and $50 \%$ coagulation.

The thicker electrode than the one that is used for skin incisions induces an extra coagulation so that the incision is almost bloodless. If vessels or possible bleeders are seen, they can be grasped with a forceps and coagulated by touching the forceps with the electrode and activating the unit shortly. We design this indirect coagulation.

\section{The Incision}

The incision in the conjunctiva is very superficial, parallel to the lower tarsal border but $2 \mathrm{~mm}$ lower. The incised conjunctiva is lifted with the forceps and then a similar incision is made through the retractor muscles. This technique procures a direct access to the inferior orbital fat without wounding the septum. This is an extra advantage since damaging the orbital septum may lead to eyelid malposition postoperatively. The Desmarres lid retractor is then removed. For maximal dilation of the wound, a wound hook with 3 blunt edges on the caudal side and a forceps in the cranial side are used.

\section{Removal of the Fat}

By pushing slightly on the globe, the fat now will prolapse. Sometimes additional membranes need to be cut before the fat pockets pop out. With the unit in the cut/coagulate mode, direct resection of the fat without the use of a clamp is possible. It is important to cut very slowly to have a maximal coagulation effect. Thicker blood vessels, when encountered, can be coagulated before cutting, in the same way as described for the conjunctival incision. Care has to be taken to excise only pure fat. Indeed: the inferior oblique muscle lies between the nasal and central fat pocket and must not be harmed $[4,5]$. Fat resection has to be very conservative: the pockets in the lower eyelid are not only caused by excess of fat but primarily by a herniation of the anatomical fat pockets. Besides: the quantity of orbital fat can diminish with age and we have to take care not to cause a 'sunken' eye. They can also be partially repositioned and fixated more nasally or over the inferior orbital rim to correct a tear trough [6-8].

\section{Final Steps}

At the end of the intervention we check the wound for possible bleeders. With a forceps the lid is pulled upwards to loosen eventual adhesions. We do not suture the conjunctiva but only put some antibiotic ointment. One gram of Tranexamin is administered intravenously and a cooling compress is put on the eye.

\section{Postoperative checks}

An ambulatory check up is done after 6 days. At this moment it is not unusual to notice some conjunctival edema. The skin can be moderately wrinkled. If wrinkles persist, a superficial peeling can be performed after a month. Only in very rare cases a (conservative) excision of skin is needed. This can be combined with a suspension of the orbicularis muscle to the periosteum of the temporal superior border of the orbit $[11,12]$. An external subciliary incision is then made in the lateral third of the lower eyelids extending into the crow's feet followed by a very conservative skin excision. Then a small excision of skin can be performed in the tail of the upper lid crease. A small piece of orbicularis is resected in the lower eyelid. A double armed polyglactin 5/0 suture is passed through the caudal part of the incised orbicularis, passed under the skin of the external cantus and recovered in the upper lid to be fixated at the periosteum of the orbital rim in the temporal superior sector. These skin incisions are closed with a few separate polypropylene $6 / 0$ sutures.

\section{Results}

Between Augsut 2015 to May 2016, 11 patients underwent esthetic blepharoplasty with a transconjunctival approach. We performed all blepharoplasties personally. Only one patient was male and 10 female, with a mean age of 52 years. All patients underwent a retroseptal approach. In 18 eyelids, there were isolated lower lid bags and without excess skin. In three eyelids with excess skin and orbital septum laxity a transconjunctival approach was preferred to avoid postoperative retraction (scleral show) [10]. In these cases we additionally performed a suspension of the orbicularis of the lower eyelid to the periosteum of the superior orbital rim.

Surgery was performed under general anesthesia conditions in all cases. The average operating time for bilateral lower eyelid transconjunctival approach was $40 \mathrm{~min}$. All patients were followed-up for at least 2 months with medical control at day 1, day 15 , and day 60 after surgery.

Moderate edema of the lower eyelid in the weeks after surgery was reported in seven patients and fast resolved. A postop limited ecchymosis has been noted in 4 patients.

The main complication of this approach is under correction of the orbital bags, due to an insufficient fat resection. That happened in three patients and concerned the nasal fat pocket in one case and the temporal fat pocket in 2 cases. The patient with the under-corrected nasal fat pocket underwent a second transconjunctival blepharoplasty through the same approach to resect some more fat. In the case of the temporal fat pocket, a transcutaneous reintervention was performed under local anesthesia. 


\section{Discussion}

Transconjunctival inferior blepharoplasty is our preferred technique for the correction of orbital bags. The transconjunctival approach allows a safe and precise excision of orbital fat. By avoiding a skin incision in the lower eyelid, there is no visible scar. Especially in younger patients with only limited wrinkles and skin folds this is an important aspect. Also the risk of retraction and scleral show is minimal.

Postoperative hemorrhage, although rare too, must be identified early. The risk of a deep or even sight threatening retrobulbar hematoma is reduced with the use of Radio Frequency surgery in the cut-coagulation mode. Also, the traction on the eyelid structures created by the Desmarres lid retractor creates a relatively bloodless surgical field. Thanks to the eversion of the eyelid a precise dissection layer by layer can be achieved avoiding a too deep incision and damaging thicker blood vessels.

Indeed, retraction of the lower eyelid can occur in patients even without skin resection, just by the incision of the skin, septum and orbicularis oculi muscle. The transconjunctival approach avoids incision of these tissues, and minimizes thus he retraction risk. In patients with excess skin, wrinkles of the lower eyelid can persist after surgery for up to three months. After 6 weeks these wrinkles can be treated with laser or superficial chemical peeling [9]. If the excess of skin is too pronounced, a minimal skin excision can be performed [10].

Entropion is exceptional after a transconjunctival approach, despite the incision of palpebral retractile tissues. We have seen a transitory postoperative diplopia in a few cases. It seemed to have been induced by the injected anesthetic. It never lasted more than $2 \mathrm{~h}$. However, prolonged and even permanent diplopia has been reported after damaging the inferior oblique and even the inferior rectus muscle $[3,4]$.

Wounding of the eyeball is always possible and should be prevented by the use of corneoscleral contact lenses.

The most common complication is undercorrection with under- excision of the orbital fat, while overcorrection is particularly rare [4]. For proper evaluation of the fat excision, the lipectomy should begin with the medial fat pocket to give easier access to the central and lateral pocket.

\section{Conclusions}

For the lower lid blepharoplasty we prefer the transconjunctival approach. It reduces the risk of postoperative eyelid retraction and scleral show. Especially in younger patients with good skin quality and only few wrinkles and skin folds, it is very attractive because there is no visible scar on the eyelid skin. Everting the lower eyelid with a Desmarres lid retractor and the use of Radio Frequency surgery in the cut/coag mode limit intra-and postoperative bleeding. The transconjunctival approach allows the reposition of orbital fat pockets to correct a tear trough and can be used in conjunction with a conservative skin resection if needed.

\section{References}

[1]. Korchia D, Braccini F, Paris J, Thomassin JM (2003) Transconjunctival approach in lower eyelid blepharoplasty. Can J Plast Surg. 11(3): 166-170.

[2]. Verity DH, Collin JR (2001) Eyelid reconstruction: the state of the art. Curr Opin Otolaryngol Head Neck Surg. 2(4): 344-348.

[3]. Pack S, Quereshy FA, Altay MA, Baur DA (2016) Transconjunctival Lower Blepharoplasty. Atlas Oral Maxillofac Surg Clin North Am. 24(2): 147-151.

[4]. Mowlavi A, Neumeister MW, Wilhelmi BJ (2002) Lower blepharoplasty using bony anatomical lasndmarks to identify and avoid injury to the inferior oblique muscle. Plast Reconstr Surg. 110(5): 1318-22; discussion 13231324 .

[5]. Morax S, Touitou V (2006) Complications of blepharoplasty. Orbit. 25(4): 303-318.

[6]. Goldberg RA (2000) Transconjunctival orbital fat repositioning: transposition of orbital fat pedicles into a subperiosteal pocket. Plast Reconstr Surg. 105(2):743-8. discussion 749-751.

[7]. Davison SP, Irio M, Oh C (2015) Transconjunctival lower lid blepharoplasty with and without fat repositioning. Clin Plast Surg. 42(1): 51-56.

[8]. Zoumalan CI, Roostaeian J (2016) Simplifying Blepharoplasty. Plast Reconstr Surg. 137(1): 196e-213e.

[9]. Bosniak S, Cantisano-Zilkha M, Purewal BK, Zdinak LA (2006) Combination therapies in oculofacial rejuvenation. Orbit. 25(4): 319-326.

[10]. Kim EM, Bucky LP (2008) Power of the pinch: pinch lower lid blepharoplasty Ann Plast Surg. 60(5): 532-537.

[11]. Bajaj MS, Pushker N, Balasubramanya R (2004) Lower eyelid dermatochalasis with Massive postural herniation of orbital fat. Orbit. 23(1): 41-44.

[12]. Lee AS, Thomas JR (2005) Lower lid blepharoplasty and canthal surgery. Facial Plast Surg Clin North Am. 13(4): 541-551. 